\title{
Light collection simulation when determining light yield of single crystal and polycrystalline organic scintillators
}

\author{
T.E.Gorbacheva ${ }^{1}$, V.A.Tarasov ${ }^{1}$, N. Z.Galunov ${ }^{1,2}$ \\ ${ }^{1}$ Institute for Scintillation Materials NAS 61001, Lenin av. 60, Kharkov, \\ Ukraine \\ ${ }^{2}$ V. N. Karazin Kharkiv National University, Svobody Sq. 4, 61022, \\ Kharkiv, Ukraine
}

Received August 2, 2015

The light-collection coefficients were calculated for different scintillation detectors using discrete optical model. Characteristics of optical media, which cannot be measured directly, were fitted using experimental response of scintillation materials with different light collection conditions. Using proposed technique absolute light yield (ALY) of single crystal, composite and polycrystalline scintillators on the base of stilbene, anthracene, pure and doped $p$-terphenyl were determined. Average ALY values obtained in this study are similar to the results for the single-crystal samples.

Keywords: composite, polycrystalline scintillators, scintillation materials, absolute light yield.

Рассчитаны коэффициенты светособирания для различных сцинтилляционных материалов с использованием дискретной оптической модели. Характеристики оптических сред, которые нельзя измерить прямыми методами, были подобраны с использованием экспериментальных откликов сцинтилляционных материалов для различных условий светособирания. Используя предложенную методику, определен абсолютный световой выход (AСB) монокристаллических, композиционных и поликристаллических образцов на основе стильбена, антрацена, чистого и активированного $n$-терфенила. Полученные усредненные значения АСB соответствуют значениям для монокристаллических образцов.

Моделювання збору світла при визначенні світового виходу в монокристалічних $\mathbf{i}$ полікристалічних органічних сцинтиляторах. Т.Є.Горбачова, В.О.Тарасов, М.З.Галунов.

Розраховано коефіцієнти збирання світла для різних сцинтиляційних матеріалів 3 використанням дискретної оптичної моделі. Характеристики оптичних середовищ, що не можна виміряти прямими методами, були підібрані з використанням експериментальних відгуків сцинтиляційних матеріалів для різних умов збирання світла. Використовуючи запропоновану методику були визначені абсолютний світловий вихід (ACB) монокристалічних, композиційних і полікристалічних зразків на основі стильбену, антрацену, чистого і активованого $n$-терфенілу. Усереднені значення ACB, які були отримані в цій роботі відповідають значенням для монокристалічних зразків.

\section{Introduction}

Scintillators based on organic single crystals of anthracene, stilbene, and p-ter- phenyl are used for detecting fast neutrons, and charged particles. These materials may be used for production of large area com- 
posite or polycrystalline scintillators. To predict the possible applications of such scintillators it is necessary to determine a number of their characteristics, the main of which is absolute light yield (ALY). Knowledge of ALY is also important for studies of the mechanism of the scintillation process and to find ways to improve their performance.

The relatively small number of ALY studies in heterogeneous scintillator is due to the difficulties of determining the optical characteristics of the scintillator and the lack of appropriate models of light-collection in such systems. Their common features are the optical heterogeneity and heterogeneity of the stopping power for ionizing radiation. This leads to a strong scattering and incomplete energy loss light scintillator particles.

Determination of ALY of scintillators bases on measurements and calculations of: "scintillator - photo-detector" system response to the absorption of a radiation of particular energy in the scintillator; photoelectron and energy calibration of this response; the quantum efficiency of the photo-detector, spectral matching factor of the emission of the scintillator and photodetector sensitivity; light collection coefficient of scintillations on the photo-detector. In [1] the published data concerning determination of ALY of a number of organic scintillators made by different authors are analyzed. Large variation on ALY was associated with: different methodological approaches to measurements, with a degree of accounting for various influencing factors, the peculiarities of calculating the light-collection in these systems. Measurements and calculations of light-collection conducted in [1], taking into account mentioned analysis, made it possible to obtain the values of ALY for single crystals of anthracene, stilbene and p-terphenyl consistent with those of other authors.

In [2], where the absolute light output of heterogeneous stilbene scintillator was estimated, light-collection ratio was determined by modeling the transmission of light in a continuous scattering medium by the Monte Carlo method. In the simulation of light transport in a such medium it is commonly adopted to utilize alternating draws of the path length and the photon scattering directions using predetermined phase function of the scattering [3]. In [2] this approach has been applied for single crystal of stilbene, polycrystalline and composite scintillators based on single crystal grains of stilbene. In the latter case the use of a continuous model of the scattering medium is not entirely correct. Composite scintillators have grains of relatively big size (up to a millimeter) with a clear optical border, on which light is scattered.

In [4] discrete model of the scintillator of columnar structure is used to simulate the light transport. This description is most suitable for composite scintillators which comprise scintillator grains surrounded by an optical medium with a different refractive index. In this case the optical characteristics of both components are usually known or can be determined experimentally.

Such description is also possible for polycrystalline scintillators. However, in this case, there is an uncertainty in the optical characteristics of the boundary layer between the grains. During compression, the deformation of the surface layers forms grain boundary media with other than grains optical characteristics. However, these characteristics can be determined during the simulation. For example, when simulating the light transport in scattering media using the iterative methods, optical parameters are varied until the calculated reflectance and transmittance values coincide with the results of measurements [5]. In the course of successive simulations such values for parameters are picked up, for example, the coefficient of volume scattering that simulated ratio of the light-collection in the samples with optical contact or without it approaches experimentally found ratio of the corresponding photo-detector responses [6].

In this work, this approach is applied to determine the coefficients of light-collection and ALY of scintillators on the base of stilbene, anthracene, pure and doped $p$-terphenyl.

\subsection{Absolute light yield and technical light output.}

The amplitude of voltage pulse upon absorption in scintillator of specific energy of ionizing radiation can be represented as:

$$
A=E \cdot N_{0} \cdot \tau \cdot k_{1} \cdot k_{2} \cdot k_{g}
$$

where $A$ is pulse amplitude at the output of the spectrometric channel, $\mathrm{mV}, E$ is the energy absorbed in the scintillator, $\mathrm{MeV}, N_{0}$ is the number of scintillation photons per absorption of energy $E=1 \mathrm{MeV}, \tau$ is a coefficient of light collection, $k_{1}$ is quantum efficiency of the photo detector, in photo- 
electrons per a photon, $k_{2}$ is a spectral matching factor between a luminescence spectrum of a scintillator and a spectral response of a photo detector, $k_{g}$ is a gain of spectrometric channel, including photo-detector preamplifier and amplifier.

The value of $N_{0}$ is the absolute light yield (ALY) of scintillator. The product of $N_{0}$ and $\tau$ gives a technical light output (TLO).

Determination of ALY is carried out in several stages. Initially TLO is measured as the number of photons $N$ per $1 \mathrm{MeV}$ of absorbed energy. On the second step lightcollection coefficient $(\tau)$ is calculated and ALY is determined as

$$
N_{0}=N / \tau \text {. }
$$

To determine the TLO the number of photons incident on a photodetector $(E \cdot N)$ must be linked to the energy absorbed in the scintillator $(E)$. There are several methods for determining TLO [7, 8]. The simplest method among those given in [7] is a relative one. The response of the test sample $(A)$ is compared to the response of standard sample $\left(A_{s s}\right)$ with a known TLO $\left(N_{s s}\right)$ under the same measurement conditions $\left(k_{1}\right.$ and $k_{g}$ ). For the scintillators with the luminescence spectrum different from the luminescence spectrum of standard the difference in the coefficients $k_{2}$ should be taken into account. Then, the TLO can be determined from the relationship

$$
N=\frac{A \cdot N_{s s} \cdot k_{2 s s}}{A_{s s} \cdot k_{2}}
$$

where $k_{2}$ is a spectral matching factor between luminescence spectrum of test scintillator and sensitivity spectrum of a photo detector; $k_{2 s s}$ is a spectral matching factor between luminescence spectrum of standard scintillator and sensitivity spectrum of a photo detector.

Light-collection coefficient $(\tau)$ is calculated on the second step either analytically or by numerical methods. Light-collection coefficients were determined by mathematical simulation of light transport in the scintillator.

\subsection{Simulation of the light transport in the heterogeneous scintillator.}

In $[9,10,15]$ heterogeneous scintillator represented as closely packed spheres with a dispersion medium filling the gaps. In the current work the whole volume of the sam- ple were divided into imaginary cells with the location of the dispersed particle of the specific shape (random size and random location). For the dispersed phase in the form of spheres one can get the volume fill factor of 0.52 (body-centered cubic packing).

The following main points were used to build algorithm of simulation of light transport in heterogeneous scintillators. The light emission is isotropic in the scintillator. It propagates according to the laws of geometrical optics. There is the probability of "reflection-transmission" on the boundaries. Fresnel laws were used to estimate the probability of "reflection-transmission" on the boundaries e.g. to describe the part of reflected energy $F_{r}(\lambda, \theta)[11]$ :

$$
F_{r}(\lambda, \theta)=\frac{\left[\frac{\cos \theta_{i}-\eta \cdot \cos \theta_{t}}{\cos \theta_{i}+\eta \cdot \cos \theta_{t}}\right]^{2}+\left[\frac{\eta \cdot \cos \theta_{i}-\cos \theta_{t}}{\eta \cdot \cos \theta_{i}+\cos \theta_{t}}\right]^{2}}{2},
$$

where $\theta_{i}$ and $\theta_{\mathrm{t}}$ are the angles of incidence and refraction, respectively; $\eta$ is a relative refractive index.

To calculate this value it is enough to know the refractive indexes of adjacent media. To estimate the probability of light absorption in the part of its path it is necessary to set the light absorption coefficient in the grains volume and in its surrounding environment.

In simulation of light-collection the intersections of light rays with both the real boundaries of the dispersed phase particles of specific shape and with the virtual cell boundaries were taken into consideration. The latter is necessary for the operation of the program cycles. Each cycle to find the intersection with the dispersed phase particle starts after entering of the beam into the new virtual cell. Once the beam enters the virtual cell, the following cases are possible (Fig. 1):

1) the ray intersects the surface of the particles and after refraction falls into it again where after a number of reflections it may be absorbed, or after refraction enter the same virtual cell, and then the following one;

2) the ray intersects the surface of the particle and reflects from it moving to the next virtual cell;

3) the ray does not cross the boundary of the particle and enters next virtual cell.

In the 1-st case the "walk" cycle of the beam inside dispersed phase particle starts with a draw of "reflection-transmission" at the boundaries. Upon reflection the cycle con- 


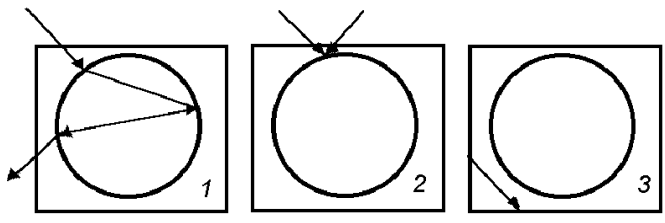

Fig. 1 Possible cases of the passage of the light beam in a virtual cell in the discrete model of heterogeneous scintillator (explanation in text).

tinues, and after the refraction the beam is sent to the next virtual cell. In cases 2 and 3 new cycle of crossing starts in the next cell.

The light-collection coefficient is defined as the ratio between counted by photo-detector and emitted rays.

To carry out simulations with this model it is necessary to set the light absorption coefficients and the refractive indices of the scintillation particles and their environment. These parameters are known for the singlecrystal scintillators and thus for grains of polycrystalline and composite scintillators.

A deformation of the surface layers, which takes place during the preparation of a polycrystalline scintillator, causes the formation of grain boundary medium with optical characteristics different from that of the grains. In our model this layer is considered to be an environment in which scintillator grains are placed. The optical characteristics of this medium are fitting parameters. They are changed iteratively until the ratios of obtained by modeling light-collection coefficients come close to the ratios of TLO found from the experimental responses in pulse amplitude spectra.

For comparison with experiment four cases of light-collection were simulated in each sample:

emergence of scintillations in the local part of the scintillator surface (with reflector - case 1 , and without it - case 2). This corresponds to the excitation of scintillations by internal conversion electrons (ICE);

emergence of scintillations evenly throughout the volume of the scintillator (with reflector - case 3 , and without it - case 4). This corresponds to the excitation of scintillations by Compton electrons those are produced by penetrating gamma radiation.

For four conditions of light collection three ratios between coefficients of the light-collection in the same sample may be calculated. This gives the ability to fit values of three adjustable parameters namely a light absorption coefficient, refractive index and an average thickness of the layer of the medium between the scintillator grains.

\section{Experimental}

For experimental studies single-crystal and polycrystalline scintillators based on anthracene, stilbene, $p$-terphenyl and $p$-terphenyl doped with 1,4-diphenyl-1,3-butadiene were used. They were $30 \mathrm{~mm}$ in diameter and $5 \mathrm{~mm}$ thick.

The single-crystal scintillators were grown from the melt by Stockbarger technique. Polycrystalline samples were made from grains with size of $2.2-2.5 \mathrm{~mm}$. The grains were prepared by crushing the corresponding single crystal ingot under a layer of liquid nitrogen. The anthracene ingot was additionally purified by zone melting [12].

The samples were obtained under the pressure of uniaxial compression of $30 \mathrm{MPa}$ and a pressing temperature of $120{ }^{\circ} \mathrm{C}$ for a stilbene, $165{ }^{\circ} \mathrm{C}$ for paraterphenyl and $140{ }^{\circ} \mathrm{C}$ for anthracene. The exposure time at this pressure and temperature was 60 minutes. After this time, the pressure was gradually reduced to atmospheric for 10 minutes and the temperature was gradually reduced to room temperature for 120 minutes.

Pulse height spectra were recorded using a scintillation spectrometer which included a studied scintillator, a photomultiplier tube (PMT) type Hamamatsu R1307, high voltage unit BCF-30, charge-sensitive preamplifier BUS2-94, BUS2-95 spectrometric amplifier, multi-channel pulse-height analyzer AMA-03F. Scintillations were excited by conversion electrons ( $E=624 \mathrm{keV})$ from $137 \mathrm{Cs}$ type OSIKE radionuclide source and Compton recoil electrons $\left(E_{\max }=477 \mathrm{keV}\right)$, which were produced in samples by gammaradiation from the same radionuclide source.

Stilbene single crystal measuring $\varnothing 30 \times 50 \mathrm{~mm}^{2}$ was used as a reference scintillator. When it was packed in a diffuse reflector omitting an exit window and with no optical contact with the PMT its technical light output was 9670 photons/MeV. The TLO of stilbene single crystal sample $30 \mathrm{~mm}$ in diameter and $5 \mathrm{~mm}$ thick measured relatively to the reference was 7990 photons $/ \mathrm{MeV}$. The end surfaces of the sample were polished, the side one - grinded. The sample was packed in a diffuse reflector with a reflection coefficient of 0.96 (three layers of Teflon tape). This sample was used as a reference for TLO calculations of all 
samples studied in this paper for both packaging options with or without a reflector.

\section{Results and discussion}

For two packaging options (with or without a reflector) of the samples pulse height spectra were recorded. Typical spectra are shown in Fig. 2.

From these spectra the positions of peaks which correspond to internal conversion electrons $(E=624 \mathrm{keV})$ and the Compton edges $\left(E_{\max }=478 \mathrm{keV}\right)$ were found.

TLOs of test samples were found by comparing the ICE peak positions and Compton edges for these samples with packed sample of stilbene single crystal $\varnothing 30 \times 5 \mathrm{~mm}^{2}$ with known TLO $(N=7990$ photons $/ \mathrm{MeV})$.

For a correct comparison with the original sample measured values of ICE peak positions were reduced to the energy of Compton edge. In the case of anthracene the positions of ICE peaks and Compton edges were further corrected taking into account the differences between its spectrum matching factors with the PMT (0.883) from that of stilbene (0.967). In the case of PTF (0.962) and doped PTF (0.966) the differences in spectral matching factors are negligible.

The positions of ICE peaks and Compton edges of test samples are shown in Table 1. It also presents the corrected positions of peaks and edges, taking into account the energy of the radiation and the spectrum matching factors between scintillators and PMT.

TLO values for samples was determined as the ratio of ICE peak or Compton edge positions according to expression (3) with respect to the test stilbene sample covered with reflector (marked in bold in Table 1 ). The TLO values are presented in Table 2 .

Table 2 also shows calculated coefficients of light-collection $\tau$ (obtained by simulation) and the value of the ALY obtained according to equation (2). Table 2 shows the results of simulation of $\tau$ for the stilbene single crystal with a reflector according to two different approaches. The model of a continuous medium the same as in [2], and the model of discrete medium is different and used in the present work.

Determination of ALY of organic singlecrystal scintillator were conducted in a number of works. In [13] it has been found that the ALY of PTF single crystals is 35000 photons $/ \mathrm{MeV}$. This value was obtained using light attenuation factors and local excitations of scintillations with alpha particles and electrons. Mentioned factors were found from experimental dependencies
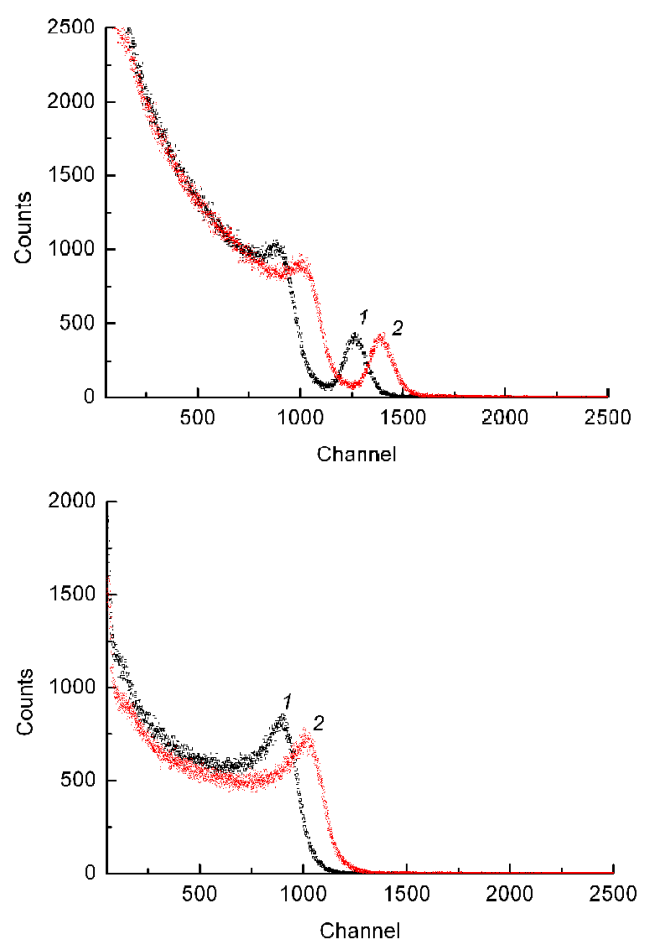

Fig 2. Typical pulse-height spectra measured with stilbene poly (1) and single (2) crystal scintillator when irradiated with a) internal conversion electrons and b) gamma rays from ${ }^{137}$ Cs source.

of scintillation light decreasing on the sample thickness. ALY was obtained by extrapolating the found dependence to the zero thickness. In this work, the attenuation factor was used instead of the light-collection coefficient. This approach leads to an overestimation of the ALY values.

In [2] ALY was investigated for single crystal, polycrystalline and composite scintillators based on stilbene. TLO was determined relatively to a standard sample of stilbene single crystal, and the light-collection coefficient - using Monte Carlo simulation. The model of a continuous scattering medium with given coefficients of absorption and scattering of light was used. The coefficients were determined from the dependence of the transmission of light of different wavelengths on the thickness of the samples. In the drawing direction of the scattering Henie-Greenstein phase function of the scattering was used [13]. For different variants of stilbene scintillator different values for TLO and close values of ALY (close to $14700 \mathrm{ph} / \mathrm{MeV}$ ) were obtained. This is because light-collection is different in a single crystal, polycrystalline and composite samples, but the light is generated in the grains with identical scintillation effi- 
Table 1. Experimental responses of single crystal and polycrystalline organic scintillators when irradiated with ICE and gamma rays from ${ }^{137} \mathrm{Cs}$ source

\begin{tabular}{|c|c|c|c|c|c|}
\hline \multirow[t]{2}{*}{$\begin{array}{c}\text { Sample } \\
\varnothing 30 \times 5 \mathrm{~mm}\end{array}$} & \multirow[t]{2}{*}{$E, \mathrm{MeV}$} & \multicolumn{4}{|c|}{$\begin{array}{l}\text { The position of peaks }(624 \mathrm{keV}) \\
\text { and the absorption edges }(478 \mathrm{keV}) \text {, channels }\end{array}$} \\
\hline & & Experiment & After correction 1) & Experiment & After correction 1) \\
\hline \multicolumn{2}{|c|}{ Stilbene } & \multicolumn{2}{|c|}{ single crystal } & \multicolumn{2}{|c|}{ polycrystalline } \\
\hline With reflector & 0.478 & 1304 & 1304 & 1170 & 1170 \\
\hline With reflector & 0.624 & 1511 & 1162 & 1182 & 905 \\
\hline Without reflector & 0.478 & 987 & 987 & 931 & 931 \\
\hline Without reflector & 0.624 & 1271 & 974 & 946 & 725 \\
\hline \multicolumn{2}{|c|}{ Anthracene } & \multicolumn{2}{|c|}{ single crystal } & \multicolumn{2}{|c|}{ polycrystalline } \\
\hline With reflector & 0.478 & 1444 & 1581 & & \\
\hline With reflector & 0.624 & 1805 & 1514 & & \\
\hline Without reflector & 0.478 & 1250 & 1369 & & \\
\hline Without reflector & 0.624 & 1631 & 1368 & & \\
\hline \multicolumn{2}{|c|}{$p$-Terphenyl } & \multicolumn{2}{|c|}{ single crystal } & \multicolumn{2}{|c|}{ polycrystalline } \\
\hline With reflector & 0.478 & 1428 & 1428 & 1314 & 1314 \\
\hline With reflector & 0.624 & 1850 & 1417 & 1157 & 886 \\
\hline Without reflector & 0.478 & 1025 & 1025 & 949 & 949 \\
\hline Without reflector & 0.624 & 1321 & 1012 & 840 & 643 \\
\hline \multicolumn{2}{|c|}{$p$-Terphenyl DPB +2$)$} & \multicolumn{2}{|c|}{ single crystal } & \multicolumn{2}{|c|}{ polycrystalline } \\
\hline With reflector & 0.478 & 1915 & 1915 & 1674 & 1674 \\
\hline With reflector & 0.624 & 2384 & 1826 & 1604 & 1229 \\
\hline Without reflector & 0.478 & 1267 & 1267 & 1304 & 1304 \\
\hline Without reflector & 0.624 & 1595 & 1222 & 1149 & 880 \\
\hline
\end{tabular}

1) for the energy of $0.624 \mathrm{MeV}$ peak position was reduced to energy of $0.478 \mathrm{MeV}$; for anthracene positions of peaks and edges were additionally reduced to the spectral matching factor of stilbene;

2) DPB - diphenylbutadiene.

ciency. This result shows the relevance of continuous optical medium model in the case of single crystal, polycrystalline and composite scintillators.

Table 2 shows that the TLO values for polycrystalline samples is lower than that for single crystals. Such dependencies exist for samples with and without a reflector. The presence of reflector smooth's dependences for single-crystal samples and enhances them for polycrystalline samples. These effects are associated with an increase in the average length of the light path to the output window both in the case of transition to polycrystalline samples, and in the case of transition from the volume to local excitation, remote from the output window.

Calculated light-collection coefficients repeat found patterns. This indicates a dependence of the TLO in this case primarily on light-collection conditions. Calculated from these data ALY values are close for samples made of identical scintillation materials. Their average values are shown in Table 3 .

It follows from Tables 2 and 3 that maximum deviations from the average values are observed for the less transparent scintillators based on anthracene single crystal $( \pm 13 \%)$ and polycrystalline p-terphenyl $( \pm 11 \%)$. For other scintillators the scatter does not exceed $10 \%$. In determining the light yield this result is acceptable [7].

Average ALY values obtained in this study are similar to the results for the single-crystal samples in [1]. On the one hand, this can be explained by the fact that the investigated samples were obtained from the same raw material using the same technology and measured in the same conditions with respect to the same reference sample. On the other hand, the possibility of application of discrete optical medium model for the determination of the coefficients of 
Table 2. Technical light output, light-collection coefficients and the absolute light yield of single gle crystal and polycrystalline organic scintillators.

\begin{tabular}{|c|c|c|c|c|c|c|c|}
\hline Sample $\varnothing 30 \times 5 \mathrm{~mm}$ & $\mathrm{E}, \mathrm{keV}$ & $\begin{array}{c}\text { TLO, ph / } \\
\mathrm{MeV}\end{array}$ & $\tau$ & $\begin{array}{c}\mathrm{ALY}, \mathrm{ph} / \\
\mathrm{MeV}\end{array}$ & $\begin{array}{c}\text { TLO, ph / } \\
\mathrm{MeV}\end{array}$ & $\tau$ & $\begin{array}{c}\mathrm{ALY}, \mathrm{ph} / \\
\mathrm{MeV}\end{array}$ \\
\hline \multicolumn{2}{|l|}{ Stilbene } & \multicolumn{3}{|c|}{ Single crystal } & \multicolumn{3}{|c|}{ Polycrystalline } \\
\hline With reflector * & 0.478 & 7990 & 0.551 & 14501 & & & \\
\hline With reflector & 0.478 & 7990 & 0.543 & 14715 & 7169 & 0.463 & 15484 \\
\hline With reflector & 0.624 & 7120 & 0.458 & 15546 & 5548 & 0,365 & 15199 \\
\hline Without reflector & 0.478 & 6048 & 0,418 & 14468 & 5705 & 0,384 & 14856 \\
\hline Without reflector & 0.624 & 5965 & 0,375 & 15908 & 4440 & 0.311 & 14277 \\
\hline \multicolumn{2}{|c|}{ Anthracene } & \multicolumn{3}{|c|}{ Single crystal } & & & \\
\hline With reflector & 0.478 & 9688 & 0,372 & 26044 & & & \\
\hline With reflector & 0.624 & 9277 & 0,325 & 28543 & & & \\
\hline Without reflector & 0.478 & 8387 & 0,266 & 31529 & & & \\
\hline Without reflector & 0.624 & 8382 & 0.248 & 33800 & & & \\
\hline \multicolumn{2}{|c|}{$p$-Terphenyl } & \multicolumn{3}{|c|}{ Single crystal } & \multicolumn{3}{|c|}{ Polycrystalline } \\
\hline With reflector & 0.478 & 8750 & 0,552 & 15851 & 8051 & 0.444 & 18133 \\
\hline With reflector & 0.624 & 8683 & 0.497 & 17471 & 5430 & 0.342 & 15878 \\
\hline Without reflector & 0.478 & 6280 & 0,422 & 14883 & 5815 & 0,372 & 15631 \\
\hline Without reflector & 0.624 & 6200 & 0.363 & 17080 & 3943 & 0,273 & 14442 \\
\hline \multicolumn{2}{|c|}{$p$-Terphenyl DPB +} & \multicolumn{3}{|c|}{ Single crystal } & \multicolumn{3}{|c|}{ Polycrystalline } \\
\hline With reflector & 0.478 & 11734 & 0,488 & 24045 & 10257 & 0,415 & 24716 \\
\hline With reflector & 0.624 & 11189 & 0,421 & 26578 & 7528 & 0.344 & 21885 \\
\hline Without reflector & 0.478 & 7763 & 0.351 & 22118 & 7990 & 0,330 & 24212 \\
\hline Without reflector & 0.624 & 7486 & 0,294 & 25463 & 5393 & 0.251 & 21485 \\
\hline
\end{tabular}

Table 3 Average values of absolute light yield of single crystal (SC) and polycrystalline (PC) organic scintillators.

\begin{tabular}{|c|c|c|c|c|c|c|c||}
\hline \multirow{2}{*}{ Scintillator } & \multicolumn{2}{|c|}{ Stilbene } & \multirow{2}{*}{ Anthracene } & \multicolumn{2}{c|}{ PTF } & \multicolumn{2}{c|}{ PTF DFB } \\
\cline { 2 - 8 } & SC & PC & SC & SC & PC & SC & PC \\
\hline Average ALY value, ph / MeV & 15200 & 15000 & 30000 & 16300 & 16000 & 24600 & 23100 \\
\hline
\end{tabular}

light-collection in single-crystal and polycrystalline scintillators is confirmed. This is also pointed by the good agreement in the results for two cases of light-collection simulations for the same single-crystal stilbene sample with a reflector (Table 2, the top two rows), carried out in this paper.

In the polycrystalline samples systematic underestimation of ALY is observed (Table 2) under the local excitation of scintillations with ICE when compared to the volume excitation by photons of gamma radiation. For single crystal samples the opposite is true. This indicates that in the used model some factors influencing the light transmission in the dispersion medium are not considered. The model that take into account light scat- tering in the volume and on the surface of grains of scintillators could be more efficient.

\section{Conclusion}

The ALY of single crystal polycrystalline scintillators based on stilbene, anthracene, $p$-terphenyl and $p$-terphenyl doped with 1,4-diphenyl-1,3-butadiene is determined. The obtained values are close to known values for single-crystal samples of stilbene, anthracene and $p$ - terphenyl.

The regularities of changes in the TLO associated with the increase in the average length of the light path to the output window, as in the case of transition to poly- 
crystalline samples, and in the case of transition from volume to a local excitation remote from the output window are established.

The possibility to use models of discrete optical medium for modeling light-collection in polycrystalline and single crystal scintillators is shown. In simulation the approach with fitting of parameters of the optical medium is used. The parameters were fitted in such way that the ratio of the light-collection coefficients in simulations of light transport in samples with different conditions of light-collection was approaching the ratio of TLO obtained from experimental response values in the pulse-height spectra.

\section{References}

1. N.Z.Galunov, O.A.Tarasenko, V.A.Tarasov, Functional materials, 20, 304 (2013).

2. N.Z. Galunov, O.A. Tarasenko, V.A. Tarasov, Functional materials, 22, 61 (2015).
3. S.A. Pistrui-Maximean, J.M. Le.tang, N. Freud, et all., Nucl.Instr.Meth., A 581, 719 (2007).

4. A. Badano, Nucl.Instr.Meth., A 508, 467 (2003).

5. V.I. Kochubey, A.N. Bashkatov. Spectroscopy of scattering media, Saratov, University Press, 2013 [in Russian].

6. V. Alenkov, O.A. Buzanov, N. Khanbekov, et all, J. Instrumental, 1 (2013).

7. E. Sysoeva, V. Tarasov, O. Zelenskaya, Nucl. Instrum. Meth.Phys.Res., A 486, 67 (2002).

8. D. Wahl, V.B. Mikhailik, H. Kraus, Nucl. Instrum. Meth.Phys.Res., A 570, 529 (2007).

9. H. H. Ross, Liquid Scintillation Counting and Organic Scintillators, Lewis, Boca Raton, 195 (1991).

10. H. Tan, T.A. DeVol, Nucl. Instr. Meth. Phys. Res., A 515, 624 (2003).

11. A.V. Shikin, L.B. Boreskov, Computer Graphics, M. Dialog-MIFI, 288 (1996).

12. N. Z. Galunov, I. V. Lazarev, A. D. Samohin. Author's Certificate p201312050.

13. M. Angelone, G. Battistoni, F. Bellini, et all, http://arxiv.org/pdf/1305.0442v1.pdf (2013).

14. D. Toublanc, Appl. Opt., 35(18), 3270 (1996). 